\title{
Is There an Association between Norton Scale and Cognitive Impairment in Hip Fractured Elderly Patients?
}

\author{
Nadya Kagansky Ilia Stambler Eliyahu Hayim Mizrahi \\ Medical Geriatric Center Shmuel Harofe, Sackler School of Medicine, Tel-Aviv University, Tel-Aviv, Israel
}

\section{Keywords}

Cognition · Hip fracture $\cdot$ Older persons $\cdot$ Norton Scale

Scores $\cdot$ Mini-Mental State Examination

\begin{abstract}
Introduction: Geriatric assessment as an integrative part of assessment is a composite of a large number of scales. Sometimes it is difficult to transfer all of them. The Norton Scale Score (NSS) assesses the degree of risk to develop bedsores. In previous studies, a correlation between Norton Scale and function was found. A correlation between Norton Scale and cognitive assessments was not evaluated yet. The aim of this study was to determine if there is an association between Norton Scale Score and cognitive impairment. This association can further facilitate geriatric assessment in frail older patients, especially in older patients with communicative difficulties. Methods: We have performed an observational cohort study which included hip fractured older patients consecutively admitted to the rehabilitation ward of the Shmuel Harofe Geriatric Medical Center. The collected data included demographic data and data on chronic illnesses. Results of cognitive status assessment (Mini-Mental State Examination - MMSE) and Norton Scale assessment were received from the computerized patients' charts. We evaluated the association between these 2 scales. Results: The study included 224 consecutive hip fracture patients with a mean age of $81.78 \pm 7.19$ years. Norton scores at admission,
\end{abstract}

karger@karger.com www.karger.com/dem

Karger $\stackrel{\text { ' }}{5}$

GOPEN ACCESS
(C) 2021 The Author(s)

Published by S. Karger AG, Basel

This is an Open Access article licensed under the Creative Common Attribution-NonCommercial-4.0 International License (CC BY-NC) (http://www.karger.com/Services/OpenAccessLicense), applicable to the online version of the article only. Usage and distribution for commercial purposes requires written permission. age, education, and previous stroke emerged as the only statistically significant parameters differing between those with cognitive decline and those without it. After adjusting for confounding variables, lower Norton scores at admission (OR 1.303, Cl: 1.097-1.548, $p=0.003$ ) were associated with an increased risk finding for cognitive impairment. Conclusion: Our findings suggest that there is an association between Norton Scale Scores and cognitive impairment. Norton score parameters, under certain circumstances, such as speech and other communication difficulties, can be used as a proxy measure for MMSE to indicate cognitive impairment. These findings can be even more helpful in the present time of "COVID-19," when we have to evaluate older patients with facial masks and other defensive suits.

(C) 2021 The Author(s)

Published by S. Karger AG, Basel

\section{Introduction}

Today, geriatric assessment is an integrative part of patient's assessment in many fields of medicine such as family medicine and internal medicine. Hip fracture is a common cause of hospitalization and disability in the elderly $[1,2]$. A significant number of rehabilitation inpatients have cognitive deficits [3]. Therefore, cognitive impairment, manifested by low scores in mental status questionnaires, traditionally has been viewed as an obstacle in geriatric rehabilitation [4] and correlated with limited func-
Correspondence to:

Nadya Kagansky, batiakag@gmail.com 
tional gains and a poor rehabilitation outcome in elderly patients [5-8]. The Mini-Mental State Examination (MMSE) is probably the most widely used standardized mental status examination of cognition. However, it was developed with a set of underlying limitations. Thus, misclassification is high for older adults who are illiterate [9]. Subsequent research has demonstrated that the MMSE is biased toward people with $<8$ th grade level of education, as they are more likely to falsely test positive for dementia [10] or mild cognitive impairment [11]. Patients with sensory or language disorders may perform poorly on the MMSE, yet they may or may not have cognitive impairment. For example, people with aphasia often score low [12] or they are screened out of studies [13]. The Norton Scale Score (NSS) is a nursing assessment tool that is generally used to predict the risk of suffering and consists of 5 domains [14]. Despite various limitations of the NSS, the tool has been evaluated in aspects other than those relating to nursing. Two studies have shown that low admission NSS are associated with higher rates of postoperative complications, increased in-hospital mortality, prolonged rehabilitation, and poor rehabilitation outcomes in elderly patients following hip fracture surgery $[15,16]$. However, none of these studies have investigated the possible use of NSS results at admission, as a possible means for a simple, quick evaluation that may be easily performed by a young physician or individual qualified nurse, to indicate cognitive impairment in this population.

The present study aimed to evaluate a possible correlation of MMSE scores and NSS and to estimate whether NSS at admission in elderly patients is associated with cognitive impairment. Such data are expected to assist in evaluating the degree of cognition of the elderly with communicative difficulties. The basic hypothesis of this study was that there is an association between Norton Scale and MMSE scores at admission. As a result, in certain medical conditions, like aphasia, Norton Scale Scores may provide us with a plausible proxy evaluation measure for the patient's cognitive condition.

\section{Materials and Methods}

\section{Patients}

This observational cohort comprised 224 geriatric patients consecutively admitted to a geriatric postacute rehabilitation ward from nearby orthopedic departments, during the period 20122017. All patients suffered a traumatic low-energy pertrochanteric (extracapsular) or subcapital (intracapsular) hip fracture, have undergone fracture fixation, and were in a stable medical condition enabling immediate active postoperative rehabilitation therapy.
The exclusion criteria comprised patients whose rehabilitation period was shorter than 7 days (assuming that the extent of rehabilitation in such a short period is limited and could distort the results) and patients with other acute disabilities such as multiple trauma, medical conditions preventing active rehabilitation (such as severe chronic lung disease necessitating constant use of oxygen and cardiac failure with functional capacity stage III-IV of NYHC), and transfer to acute care departments due to complications and/or death. These criteria excluded patients with either medical or functional conditions, which would have limited their rehabilitation potential. Patients who suffered from delirium were not accepted to our rehabilitation department (they underwent rehabilitation in our acute department), and thus they were not included in the study. Complete medical details were extracted from each patient's medical chart. The study was approved by the local ethics committee.

\section{Cognitive Assessment}

All patients were evaluated for cognitive level with the MiniMental State Examination (MMSE) [17] within 1 week after admission to the rehabilitation ward, and scores $<24$ points (low MMSE) were considered indicative for a cognitive impairment while MMSE $\geq 24$ indicates nonimpaired cognitive ability [10].

\section{Norton Scale Score}

NSS is a simple scoring system that includes an assessment of general physical condition, mental status, activity level, mobility, and incontinence. It was administered by the nursing staff at admission and discharge, with 1 (minimum) and 4 (maximum) points, per each of its domains, and with a final score ranging between 5 and 20 points [14].

\section{Statistical Methods}

Comparisons between patients with and without cognitive impairment were undertaken on a list of clinical and demographic measures using paired $t$ tests for continuous variables and $\chi^{2}$ tests for dichotomous variables. Linear regression analysis and binary logistic regression were performed to study simultaneously the independent relationship between demographic and clinical parameters, cognitive impairment, and Norton scores at admission.

The statistical significance level was set to 0.05. All statistical analyses were performed using the SPSS system for Windows, version 25 (IBM Inc.). Power analysis was performed post hoc to validate the findings based on the sample $N$ and effect size using the $G^{*}$ Power application, version 3.1.9.2.

\section{Results}

The data on 224 consecutive hip fracture patients admitted to a geriatric rehabilitation ward during a 5-year period were available. The mean age was $81.78 \pm 7.19$ years, and more than half of the patients were females (62.1\%). Low MMSE values were seen in 163 patients (72.8\%). Background and clinical characteristics of all the patients are presented in Table 1. The patients were also divided according to the cognitive status with and without cognitive decline (MMSE $<24$ and MMSE $\geq 24$ ). Nor- 
Table 1. Clinical and demographic characteristics of patients by cognitive impairment (MMSE <24)
Fig. 1. The association between Norton Scale Scores and MMSE at admission (Spearman correlation coefficient $=0.486$, $p<0.001)$. MMSE, Mini-Mental State Examination.

\begin{tabular}{lllll}
\hline Variable & All & $\begin{array}{l}\text { Patients with } \\
\text { MMSE } \geq 24\end{array}$ & $\begin{array}{l}\text { Patients with } \\
\text { MMSE }<24\end{array}$ & $p$ value* \\
\hline$n$ & 224 & 61 & 163 & \\
Norton admission & $16.96 \pm 2.36$ & $18.18 \pm 1.53$ & $16.5 \pm 2.46$ & $<0.001$ \\
Age, years & $81.78 \pm 7.19$ & $78.74 \pm 7.52$ & $82.92 \pm 6.75$ & $<0.001$ \\
Education & $9.12 \pm 5.18$ & $11.7 \pm 3.7$ & $8.09 \pm 5.34$ & $<0.001$ \\
Female gender & $139(62.1)$ & $44(72.1)$ & $95(58.3)$ & 0.057 \\
Diabetes mellitus & $79(35.3)$ & $21(34.4)$ & $58(35.8)$ & 0.85 \\
Hypertension & $169(75.4)$ & $125(76.7)$ & $44(72.1)$ & 0.48 \\
Ischemic heart disease & $123(54.9)$ & $32(52.5)$ & $91(55.8)$ & 0.65 \\
Previous stroke & $32(14.3)$ & $4(6.6)$ & $28(17.2)$ & 0.043 \\
Parkinson's disease & $16(7.1)$ & $3(4.9)$ & $13(8)$ & 0.43 \\
\hline
\end{tabular}

* $p$ calculated using the $x^{2}$ test for categorical variables and Student's $t$ test for comparisons of continuous variables. MMSE, Mini-Mental State Examination.

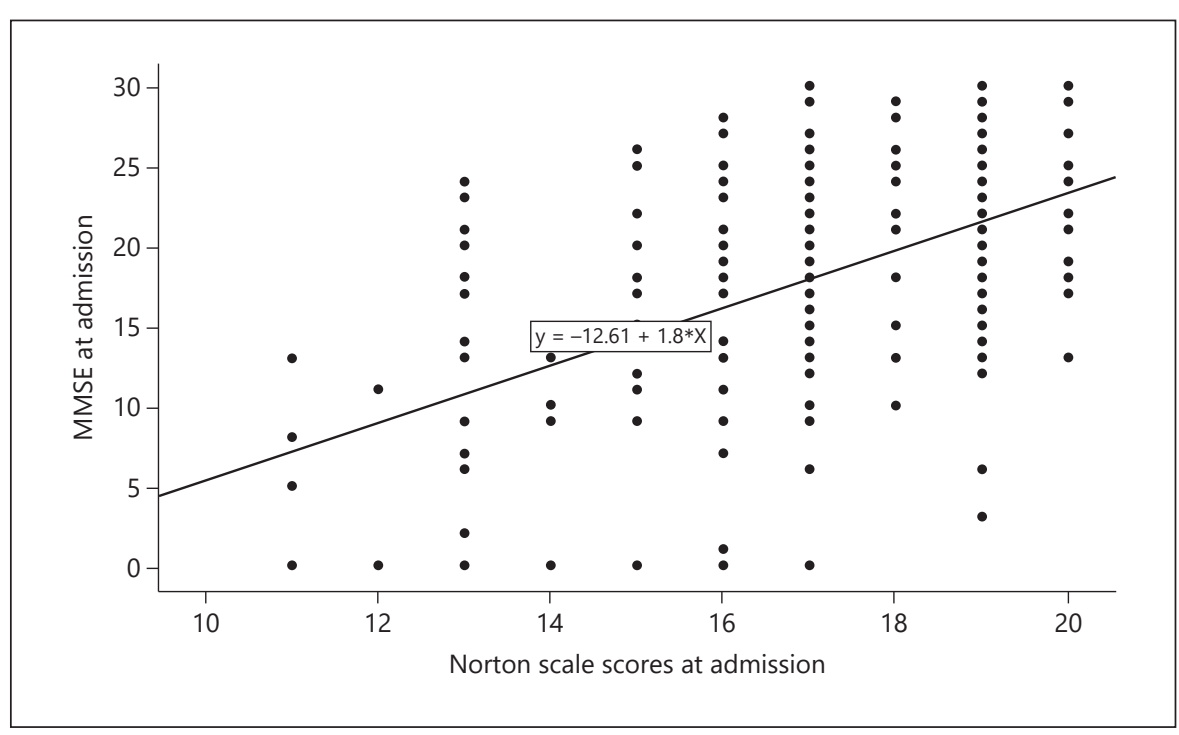

ton Scale Scores at admission, age, education, and the history of previous stroke emerged as significantly differing between these 2 groups. However, cardiovascular risk factors, such as diabetes mellitus, hypertension, and even ischemic heart disease, were not significantly different between patients with and without cognitive impairment.

Of the total 224 patients, 58 had been diagnosed with dementia prior to hospitalization in our ward. The average NSS of patients without dementia and dementia was $17.6+18.81$ and $15.12+2.64$, respectively, $p<0.001$.

Significant positive correlation was found between NSS and MMSE (Spearman correlation coefficient = $0.486, p<0.001$ ) (Fig. 1). Since patients with intact MMSE were slightly younger, we used a linear regression model in order to identify independent predictors of MMSE scores at admission. MMSE scores at admission were sig- nificantly associated with age, NSS at admission, and education $\left(R^{2}=0.33, F=11.0, \mathrm{df}=9.203, p<0.001\right)(\mathrm{Ta}-$ ble 2 ). None of the other variables tested were predictive of MMSE scores at admission.

In addition, logistic regression analysis correlating for cognitive impairment (MMSE <24) while controlling for confounding variables showed that lower Norton scores at admission (OR 1.303, CI: 1.097-1.548, $p=0.003$ ), older age (OR 0.932, CI: 0.886-0.98, $p=0.006$ ), and fewer years of education (OR 1.183, CI: 1.09-1.283, $p<0.001$ ) were all associated with an increased risk of cognitive impairment (Table 3). We also performed logistic regression analysis correlating for cognitive impairment (MMSE <18) while controlling for confounding variables, which showed that lower Norton scores at admission (OR 1.472, CI: 1.258-1.724, $p<0.001$ ) and fewer 
years of education (OR 1.118, CI: 1.0048-1.192, $p=$ $0.001)$ were all associated with an increased risk of cognitive impairment (Table 4). The power analysis of the abovementioned findings indicated very high $1-\beta$ values, approaching $p=1.0$.

Table 2. Regression coefficients of clinical variables and MMSE scores

\begin{tabular}{llc}
\hline Variable & $\beta$ & $p$ value \\
\hline Norton admission & 0.38 & $<0.001$ \\
Age (years) & -0.147 & 0.02 \\
Gender (female) & -0.004 & 0.955 \\
Education (years) & 0.26 & $<0.001$ \\
Ischemic heart disease & 0.06 & 0.413 \\
Arterial hypertension & -0.036 & 0.583 \\
Diabetes mellitus & 0.016 & 0.83 \\
Parkinson's disease & -0.015 & 0.804 \\
Previous stroke & -0.083 & 0.176 \\
\hline
\end{tabular}

MMSE, Mini-Mental State Examination.

\section{Discussion}

Clinical assessment of the older persons includes a large number of investigations, the so-called scales. Sometimes it is difficult to transfer all the scales in frail older persons. These difficulties of geriatric assessment are even more pressing in the present time of "COVID-19," when we have to evaluate older patients with facial masks and other defensive suits. Verbal contact becomes difficult and in many cases useless. Therefore, it is important to find associations between different scales and to attempt in some cases to diminish the number of transferred scales. Although low Norton Scale Scores are associated with worse physical condition, cognitive domains in the Norton Scale are more related to consciousness status, consequently to the definition of delirium, and not to other domains of cognitive assessment [14]. Thus, in their study, Justo et al. [18] found that admission ANSS may be used not only for evaluating pressure ulcer risk but also for predicting long-term mortality, i.e., within 1 year or more, following rehabilitation in older patients. In their another
Table 3. Coefficients of studied variables and cognitive impairment (MMSE <24)
Table 4. Coefficients of studied variables and cognitive impairment (MMSE <18)

\begin{tabular}{llllr}
\hline Independent predictors & $\beta$ & Odds ratio & $95 \% \mathrm{Cl}$ & $p$ value \\
\hline Norton admission & 0.265 & 1.303 & $1.097-1.548$ & 0.003 \\
Age & -0.071 & 0.932 & $0.886-0.98$ & 0.006 \\
Gender & -0.771 & 0.463 & $0.211-1.017$ & 0.055 \\
Education & 0.168 & 1.183 & $1.09-1.283$ & $<0.001$ \\
Ischemic heart disease & -0.225 & 0.799 & $0.346-1.847$ & 0.599 \\
Arterial hypertension & 0.045 & 1.0046 & $0.441-2.482$ & 0.919 \\
Diabetes mellitus & 0.245 & 1.278 & $0.517-3.156$ & 0.595 \\
Parkinson disease & 0.54 & 1.716 & $0.354-8.321$ & 0.503 \\
Previous stroke & 1.097 & 2.994 & $0.875-10.251$ & 0.081 \\
\hline
\end{tabular}

MMSE, Mini-Mental State Examination.

\begin{tabular}{llllr}
\hline Independent predictors & $\beta$ & Odds ratio & $95 \% \mathrm{Cl}$ & $p$ value \\
\hline Norton admission & 0.387 & 1.472 & $1.258-1.724$ & $<0.001$ \\
Age & -0.035 & 0.966 & $0.918-1.017$ & 0.183 \\
Gender & 0.146 & 1.157 & $0.569-2.353$ & 0.686 \\
Education & 0.111 & 1.118 & $1.048-1.192$ & 0.001 \\
Ischemic heart disease & 0.236 & 1.266 & $0.562-2.853$ & 0.569 \\
Arterial hypertension & -0.656 & 0.519 & $0.225-1.197$ & 0.124 \\
Diabetes mellitus & -0.186 & 0.83 & $0.36-1.913$ & 0.662 \\
Parkinson disease & -0.583 & 0.558 & $0.138-2.266$ & 0.415 \\
Previous stroke & 0.591 & 1.806 & $0.716-4.558$ & 0.211 \\
\hline
\end{tabular}

MMSE, Mini-Mental State Examination. 
study, they found that the Norton Scale may be used for predicting hospitalization length, complications during hospitalization other than pressure ulcers, and in-hospital mortality in older patients admitted to an internal medicine department [19]. However, we have not found a study that examines the relationships between NSS and cognitive examination.

The present study focused on the possible interrelation between NSS and cognitive impairment in a group of the older hip fracture patients. Our data show that lower NSS [14] were associated with lower MMSE scores [17] thus indicating cognitive impairment. After multivariate adjustment (for age, gender, education, diabetes, ischemic heart disease, hypertension, Parkinson's disease, and previous stroke), the association between NSS and cognitive decline remained evident. To the best of our knowledge, this is the first study that discusses this issue. Importantly, the power analysis of the abovementioned findings indicated very high 1- $\beta$ values, approaching $p=1.0$. This association between MMSE and NSS may be explained by the fact that some of the parameters being evaluated by NSS, such as urine incontinence, ability to walk, and physical conditions, can be influenced by the patient's cognitive abilities. Thus, in an observation of 6,361 communitydwelling women aged 65 years and older, it was found that both cognitive and physical functional declines are likely important contributors to urinary incontinence [20]. In a study of 4,000 community-dwelling Chinese elderly, aged 65 and older, it was shown that the cognitively impaired participants had weaker grip strength and performed worse in walk and chair stand physical function tests [21]. Furthermore, a cross-sectional study of 69 patients showed that usually walking speed is reduced in a graded fashion with the early symptoms of cognitive impairment [22]. It is important to emphasize that the MMSE, which is widely used as a standard of mental status and cognition of older patients, is not reliable among those who are illiterate or do not speak fluently the language of the administered examination $[9,10,23]$. In addition, any sensory disorder can bias the MMSE results [12, 24].

At the same time, most if not all the NSS parameters can be obtained only by observing the patients, without the need to communicate with them. Our results are in line with the hypothesis that NSS might be used for prognostication of older hip fracture patients $[15,24$, $25]$ as it comprises some basic health aspects of the older population. In addition, the use of NSS may help in overcoming the increasing need associated with the chronic shortage of professional workers in the field of geriatric care services, in particular in suburban areas, even in developed countries, where such shortage is common [26-30]. Unlike the NSS, the administration of MMSE is time-consuming taking approximately 15 min to complete. Being a much simpler, shorter, reliable, and feasible test, the NSS may serve as a proxy for MMSE to indicate cognitive impairment for this population. Clearly, the MMSE score is a much more sensitive tool for identifying cognitive impairment compared to NSS. However, the purpose of this study was to investigate whether NSS may be associated with cognitive impairment in this population.

Our study has a number of limitations resulting from its retrospective nature, being performed in a single medical center, and the study did not compare the reliability of NSS versus MMSE, but investigated the correlation of NSS with cognitive impairment as evaluated by MMSE. Despite these limitations, the present research setting is advantageous, as it has been carried out in a medical ward where all the patients are treated according to a standard rehabilitation program. We conclude that the Norton score at admission, under certain circumstances, such as among patients with speech difficulties or dementia, can be used as a proxy measure for MMSE to indicate cognitive impairment.

\section{Conclusion}

The finding of the association between Norton Scale Scores and cognitive impairment at admission among adult hip fracture patients may suggest the possibility that under certain circumstances that make it difficult to perform a direct and thorough cognitive evaluation of geriatric patients, the Norton scores at admission might serve as a proxy measure to indicate cognitive impairment. These findings can be even more helpful in the present time of "COVID-19," when we have to evaluate older patients with facial masks and other defensive suits.

\section{Statement of Ethics}

The study protocol was approved by the institute's committee on human research (Shmuel Harofe Medical Center, Beer Yaakov, Israel). An informed consent was not required, as it was an historical study based on computerized data.

\section{Conflict of Interest Statement}

The authors declare no conflicts of interest. 


\section{Funding Sources}

There was no financial support for this study.

\section{Author Contributions}

All the authors have contributed significantly to the research and manuscript, and all the authors are in agreement with the content of the manuscript. Nadya Kagansky, MD, MHA, contributed to study concept and design, analysis and interpretation of data, and preparation of the manuscript. Ilia Stambler, $\mathrm{PhD}$, contrib- uted to acquisition of subjects and data and preparation of the manuscript. Prof. Eliyahu Hayim Mizrahi, MHA, contributed to study concept and design, analysis and interpretation of data, and preparation of the manuscript.

\section{Data Availability Statement}

All data generated or analyzed during this study are included in this article. Further enquiries can be directed to the corresponding author.

\section{References}

1 Gillespie WJ. Extracts from "clinical evidence": hip fracture. BMJ. 2001;322(7292): 968-75.

2 Brainsky A, Glick H, Lydick E, Epstein R, Fox $\mathrm{KM}$, Hawkes W, et al. The economic cost of hip fracture in community dwelling older adults: a prospective study. J Am Geriatr Soc. 1997;45(3):281-7.

3 Luxenberg JS, Feigenbaum LZ. Cognitive impairment on a rehabilitation service. Arch Phys Med Rehabil. 1986;67(11):796-8.

4 Denvenney E, Hodges JR. The mini mental state examination: pitfalls and limitations. Pract Neurol. 2017;17(1):79-80.

5 Baker BR, Duckworth T, Wilkes E. Mental state and other prognostic factors in femoral fractures of the elderly. J R Coll Gen Pract. 1978;28(194):557-9.

6 Billig N, Ahmed SW, Kenmore PI. Hip fracture, depression, and cognitive impairment: a follow-up study. Orthop Rev 1988;17:315-20.

7 Magaziner J, Simonsick EM, Kashner TM, Hebel JR, Kenzora JE. Predictors of functional recovery one year following hospital discharge for hip fracture: a prospective study. J Gerontol. 1990;45(3):M101-7.

8 Rubenstein LZ, Wieland D, English P, Josephson K, Sayre JA, Abrass IB. The Sepulveda VA geriatric evaluation unit: data on four-year outcomes and predictors of improved patient outcomes. J Am Geriatr Soc. 1984;32(7):50312.

9 Scazufca M, Almeida OP, Vallada HP, Tasse WA, Menezes PR. Limitations of the minimental state examination for screening dementia in a community with low socioeconomic status: results from the sao paulo ageing \& health study. Eur Arch Psychiatry Clin Neurosci. 2009;259(1):8-15.

10 Tombaugh TN, McIntyre NJ. The mini-mental state examination: a comprehensive review. J Am Geriatr Soc. 1992;40(9):922-35.

11 Crum, RM, Anthony JC, Bassett SS, Folstein MF. Population-based norms for the minimental state examination by age and educational level. JAMA. 1993;269(18):2386-91.

12 Chow TW, Hynan LS, Lipton AM. MMSE scores decline at a greater rate in frontotem- poral degeneration than in AD. Dement Geriatr Cogn Disord. 2006;22(3):194-9.

13 D’Alessandro R, Pandolfo G, Azzimondi G, Feruglio FS. Prevalence of dementia among elderly people in Troina, Sicily. Eur J Epidemiol. 1996;12(6):595-9.

14 Norton D, McLaren R, Exton-Smith AN. An investigation of geriatric nursing problems in the hospital 1962. London, UK: National Corporation for the Care of Old People; 1962.

15 Gold A, Sever R, Lerman Y, Salai M, Justo D. Admission Norton scale scores (ANSS) and postoperative complications following hip fracture surgery in the elderly. Arch Gerontol Geriatr. 2012;55(1):173-6.

16 Justo D, Vislapu N, Shvedov V, Fickte M, Danylesko A, Kimelman P, et al. Admission Norton scale scores (ANSS) correlate with rehabilitation outcome and length in elderly patients following hip arthroplasty. Arch Gerontologoy Geriatr. 2011;53(1):e33-e36.

17 Folstein M, Folstein S, McHugh PR. Minimental state examination. A practical method for grading the cognitive state of patients for the clinician. J Psychiatr Res. 1975;12(3):18998.

18 Justo D, Guy N, Halperin E, Lerman Y, Admission Norton scale scores are associated with long-term mortality following rehabilitation in older adults. J Rehabil Med. 2012;44: 172-5.

19 Leshem-Rubinow E, Vaknin A, Sherman S, Justo D. Norton scale, hospitalization length, complications, and mortality in elderly patients admitted to internal medicine departments. Gerontology. 2013;59(6):50713.

20 Huang AJ, Brown JS, Thom DH, Fink HA, Yaffe K; Study of Osteoporotic Fractures Research Group. Urinary incontinence in older community-dwelling women: the role of cognitive and physical function decline. Obstet Gynecol. 2007;109:909-16.

21 Auyeung TW, Kwok T, Lee J, Leung PC, Leung J, Woo J. Functional decline in cognitive impairment: the relationship between physical and cognitive function. Neuroepidemiology. 2008;31:167-73.
22 Knapstad MK, Steihaug OM, Aaslund MK, Nakling A, Naterstad IF, Fladby T, et al. Reduced walking speed in subjective and mild cognitive impairment: a cross-sectional study. J Geriatr Phys Ther. 2019;42(3):E122E128.

23 Tiwari SC, Tripathi RK, Kumar A. Applicability of the mini-mental state examination (MMSE) and the Hindi mental state examination (HMSE) to the urban elderly in India: a pilot study. Int Psychogeriatr. 2009;21(1): $123-8$.

24 Foreman MD, Fletcher K, Mion LC, Simon L, Niche F. Assessing cognitive function: the complexities of assessment of an individual's cognitive status are important in making an accurate and comprehensive evaluation. Geriatr Nurs. 1996;17(5):22832.

25 Halperin E, Engel T, Sherman S, Justo D. Low admission Norton scale scores are associated with falls long after rehabilitation in the elderly with hip fractures. Clin Interv Aging. 2012;7:431-6.

26 Bo W, Hong D, Xuezong L, Zhongxin X. The demand for rehabilitation therapists in Beijing health organizations over the next five years. Disabil Rehabil. 2008;30(5):37580.

27 Landry MD, Ricketts TC, Verrier MC. The precarious supply of physical therapists across Canada: exploring national trends in health human resources (1991 to 2005). Hum Resour Health. 2007;5:23.

28 Stanmore E, Waterman H. Crossing professional and organizational boundaries: the implementation of generic rehabilitation assistants within three organizations in the northwest of England. Disabil Rehabil. 2007;29(9): 751-9.

29 Wilson RD, Lewis SA, Murray PK. Trends in the rehabilitation therapist workforce in underserved areas: 1980-2000. J Rural Health. 2009;25(1):26-32.

30 O'Toole K, Schoo AM. Retention policies for allied health professionals in rural areas: a survey of private practitioners. Rural and Remote Health. 2010;10(2):1331. 